\title{
Application of Hyperspectral Band Elimination Technique to PVT Images of Composite Structures
}

\author{
Mahmoud Zaki Iskandarani \\ Department of Computer Science, Faculty of Science and Information Technology, \\ Al-Zaytoonah University of Jordan, Amman, Jordan \\ Email: m_iskandarani@yahoo.com
}

Received July 20, 2012; revised August 22, 2012; accepted September 2, 2012

\begin{abstract}
A new approach to NDT of composite structures using Band Elimination of the analyzed image index by Hyperspectral image analysis approach is presented and discussed. The matrix Band Elimination technique allows the monitoring and analysis of a components structure based on Filtering of bands and correlation between sequentially pulsed thermal images and their indices. The technique produces several matrices resulting from frame deviation and pixel redistribution calculations for intelligent classification and property prediction. The obtained results proved the technique to be capable of identifying damaged components with ability to model various types of damage under different conditions.
\end{abstract}

Keywords: Hyperspectral; Thermography; NDE; Composites; Damage Detection; PVT; Neural Networks

\section{Introduction}

Layered composites are among the most widespread advanced materials in use. Fiber reinforced composites provide unlimited alternatives, where the performance of the whole is superior to the sum of the parts.

The light weight, very strong and stiff fibers combined with weak and brittle matrix can provide a wide range of material combinations with an opportunity for optimization that goes beyond mechanical properties to thermal, acoustic, electromagnetic characteristics.

The structural design and maintenance of composite structures involving these materials need comprehensive evaluation and characterization of mechanical properties and behavior under different loading conditions, in both undamaged and damaged states.

The complex nature of fiber reinforced composites makes them particularly difficult to test for defects. The use of composites in demanding applications like automotive and aerospace industries means, however, that it is particularly important to find the best testing methods. The increase use of these materials is expected to continue because they offer the designer, amongst other things, high specific strength and stiffness, increased design flexibility and excellent fatigue resistance.

As composite materials are finding increasing use in more demanding applications, requiring a high degree of accuracy and reliability, considerable effort is being made to define and setup quality control procedures and inspection methods.
A composite structure may also be damaged in service due to physical damage such as impact or fatigue creep. The influence of the environment on a structure due to ingress or moisture, exposure to hot and wet conditions for over long periods, contamination from oils or fluids may also cause damage, like surface abrasion and dents, delamination, fiber crack, bonding failure.

Such defects are all potentially detrimental to the mechanical integrity, and consequently to the structural performance of a component. The extent to which a defect will affect the performance will depend on the geometry of the structure, the location and orientation of the defect, the type of applied stress field and the working environment.

Confidence in the application of safety critical structural composites would be improved if a fast accurate method of assessing manufacturing flaws and service damage in relation to the structural engineering performance was available. A perceived problem with composite structures is that internal damage may seriously weaken a structure yet be undetected due to little surface evidence [1-2].

Structural damage of composites as a result of impact is regarded as one of the most critical aspects that restrict wider applications. In composites, the possibility of plastic deformation is limited which can lead to substantial amount of deformation upon impact.

At impact, a stress field is established within the composite structure, releasing a series of stress waves propagating through the material which initiates number of 
damage mechanisms that could cause splitting, de-bonding, matrix cracking, fiber pull-out, fiber breakage and delamination. The extent to which a specific failure mechanism affects the structure depends on the fiber, matrix and their inter-phase properties as well as the geometric form and fiber arrangements.

Impact is a key issue in the design of composite structures where the impact event and extent are of importance. Damage occurs progressively during an impact and is a function of the impact event and structure resistance that is affected by material properties. Local and global effects need to be considered which gives an indication regarding the structure dynamic response. Method of impacting is also a factor where supported frames respond differently to impact compared to unsupported ones, indicating that boundary conditions significantly affect structure response and extent of damage.

The application of an impact can result in a dynamic stress which when established can induce a damage that propagates at a number of sites within the material thickness. Composites with their low transverse tensile strength can be prone to this type of effect $[3,4]$.

\section{Background}

Pulse Video Thermography (PVT) is a versatile NDT inspection method, ready to be used in industrial applications assisted by computer and intelligent software specifically designed for this purpose. The subjected material reacts as a result of applying thermal pulse and propagates, by diffusion, under the surface with radiation and convection losses. The presence of a defect reduces the diffusion rate so that when observing the surface temperature, defects appear as areas of different temperatures with respect to surrounding sound areas, once the thermal front has reached them. Consequently, deeper defects will be observed later and with a reduced contrast.

In fact, the observation time $t$ is function (in a first approximation) of the squared of the depth $z$ and the loss of thermal contrast $c$ is proportional to the cube of the depth:

$$
t \approx \frac{z^{2}}{\propto} \text { and } c \approx \frac{1}{z^{3}}
$$

where $\alpha$ is the thermal diffusivity of the material.

Knowledge of the evolution of thermal contrast above the defect in conjunction with equations derived from inverse heat transfer modeling allows retrieving defect parameters such as depth, diameter, and thermal resistance. A common definition of the thermal contrast $C$ is:

$$
C(T)=\frac{T_{i}(t)-T_{i}\left(t_{0}\right)}{T_{s}(T)-T_{s}\left(t_{0}\right)}
$$

where $T$ is the temperature signal, $t$ is the time variable, subscripts $i$ and $s$ refer respectively to over a suspected defective location (that is in fact any pixel in the image) and over sound areas respectively. $C$ is computed with respect to before heating temperature distribution at time $t_{0}$ (to suppress the adverse contributions from the surrounding environment) and normalized by the behavior of a sound area so that a unit value is obtained over a non defect area.

Based on the previous, it is important to determine boundary profile to enable detection of any changes in the component under test which will result in boundary profile changes.

The sample is modeled as a two dimensional surface. A certain portion of the surface is considered to be accessible for measurements and the remainder of the surface, which may or may not be damaged to be inaccessible. It is further assumed that data can be obtained at every point on the accessible portion of the object's surface $[5,6]$.

The surface temperature of a considered component is inspected for identification of regions where heat flow is modified by subsurface anomalies. To achieve this, the component is thermally excited so that heat will flow from one face to the opposite one. The conduction of heat from the sample surface to the cooler interior is derived from the changes in the surface temperature that are detected with different rate of cooling where anomalies exist. Figure 1 illustrate the PVT process.

Such kind of analysis is common in the automotive industry. Other common applications of the active PVT scheme are in quantitative subsurface defect assessment (cracks, delaminations, impact damages, disbondings, moisture), thermophysical property evaluation; in all kind of industries [7-10].

In Hyperspectral image analysis approach, image fusion which is the process of combining relevant information from two or more images into a single image takes place. The resulting image will be more informative than any of the input images.

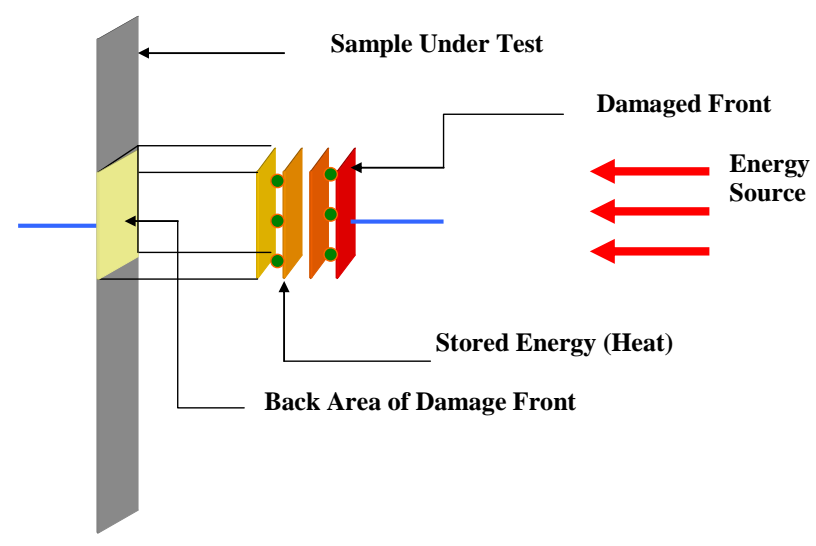

Figure 1. PVT process. 
The image fusion techniques allow the integration of different information sources. The fused image can have complementary spatial and spectral resolution characteristics. Such techniques are usually used in satellite imaging; where two types of images are available. The panchromatic image acquired by satellites is transmitted with the maximum resolution available and the Multispectral data are transmitted with coarser resolution. This will usually be two or four times lower. At the receiving end, the panchromatic image is merged with the Multispectral data to convey more information.

Despite the fact that Hyperspectral image analysis is used to perform index analysis in Hyperspectral and Multispectral satellite imagery, it is assumed that it can be used for image comparison of similar or processed images, of completely different origin [11-13].

As Hyperspectral technique uses different bands with different wavelengths to analyze the image results, which enables better recognition and classification. This should result in a successful recognition and component classification approach with characterization of tested composite [14-16].

In this paper an investigation into a possible Hyperspectral based NDT systems and algorithms that allow engineers and researchers to pinpoint defects. The system is based on incident wavelengths on a composite surface being detected and analyzed assuming the high resolution panachromatic image (PAN) to be the reference image while the image of the damaged component to be the low resolution Multispectral image (MS), as any damage in the composite causes a change in the detected wavelengths as a result of wave diffusion.

The proposed technique is suitable for high volume monitoring and inspection of safety critical components non-destructively. It unifies through conversion the extracted information from irrelevant background and using a Neural Network Integrity Classification Algorithm with the ability to correlate obtained data to level of damage and it is effect on the structure overall performance.

\section{Experimental Arrangements}

Figure 2 shows the experimental setup for impacting and testing composite structures, while, Figure 3 shows the used testing system using PVT with Hyperspectral based intelligent classification system.

An algorithm is used to compute four Hyperspectral indices. The purpose of the used program is to provide such index results in a matrix format and apply matrix analysis techniques to indentify and classify composites. Figures 4(a)-(d) show the tested RIM composite structure thermal images.

\section{Analysis and Discussion}

Three bands used to analyze the captured thermal images

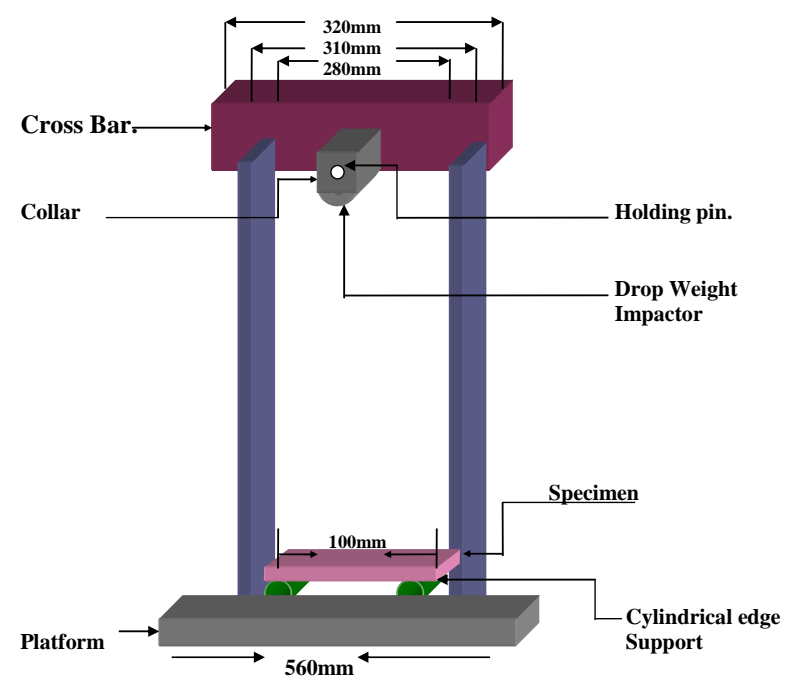

Figure 2. Impact damage system.
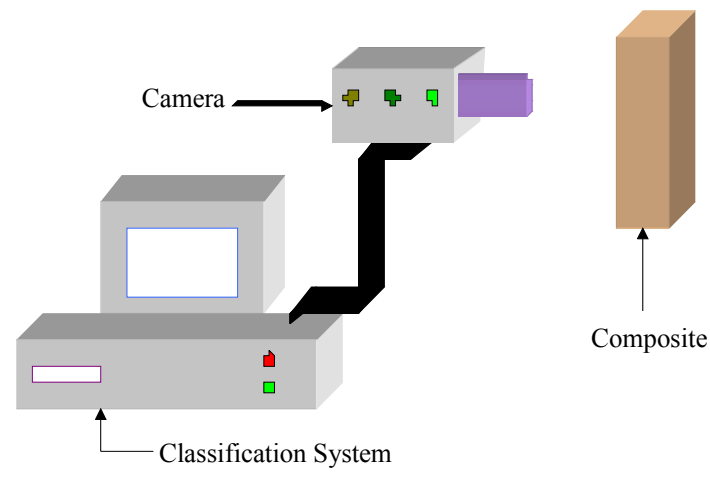

Figure 3. Testing system.

with four classifying image related functions were applied to compute image indices. Matrices (1)-(4) show results for tested component structure.

To enable selection of the best band which gives better information and classification, a principle is adopted to narrow the search space to include only the bands that the various unsupervised methods respectively deem most informative and least redundant. In this work the condition applied is that the best representative band is the one which has no negative values with uniform and smooth transitions. Hence, a characteristic matrix can now be produced per tested side per selected band. The first band is selected based on the following criteria:

As the incident energy pulse diffuses over time the subsequent indices per band should show an increasing RMSE, RASE, Deviation with decreasing Correlation values. This is due to wave propagation, which when diffused and partly captured by a damaged area (energy Trap) results in a less resembling image to the reference one as time progresses.

Hence, a final characteristic matrix could be produced to indicate the type of damage and material being tested. 


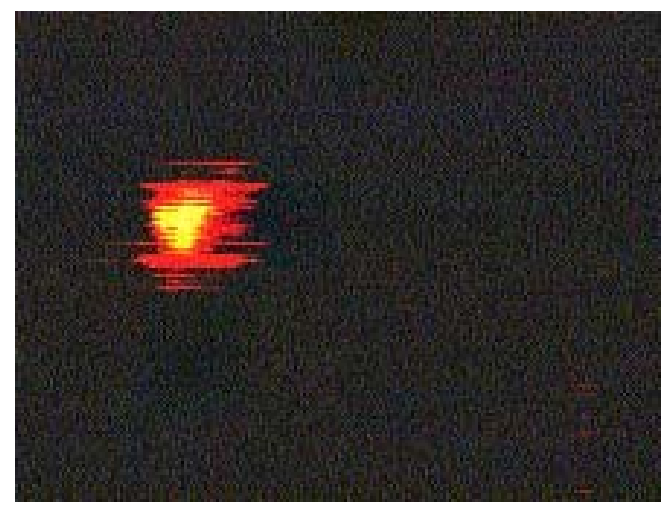

(a)

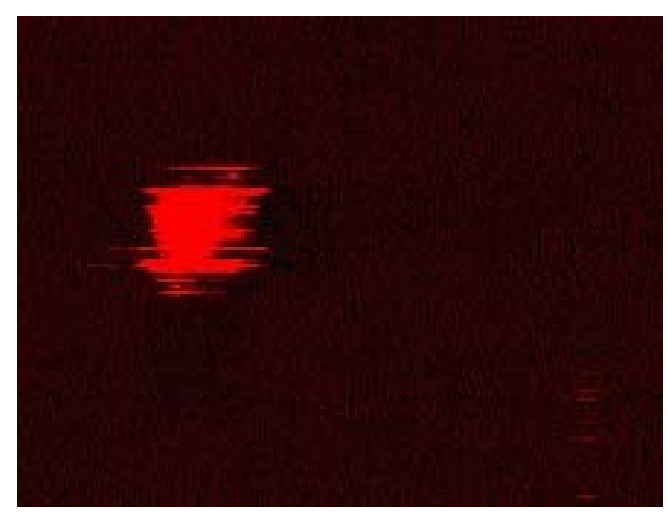

(b)

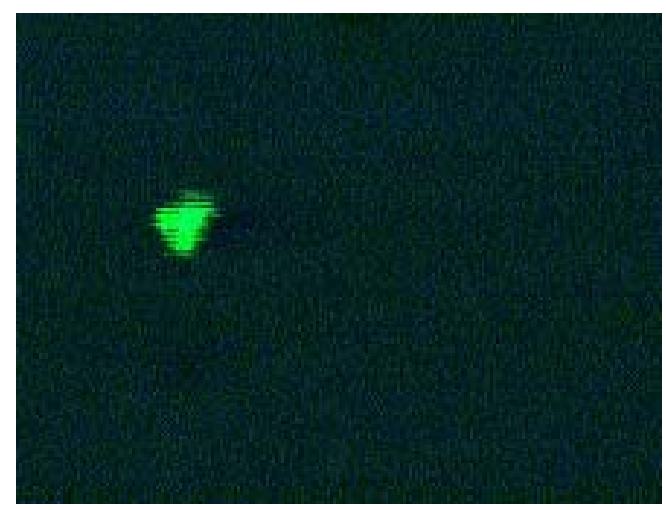

(c)

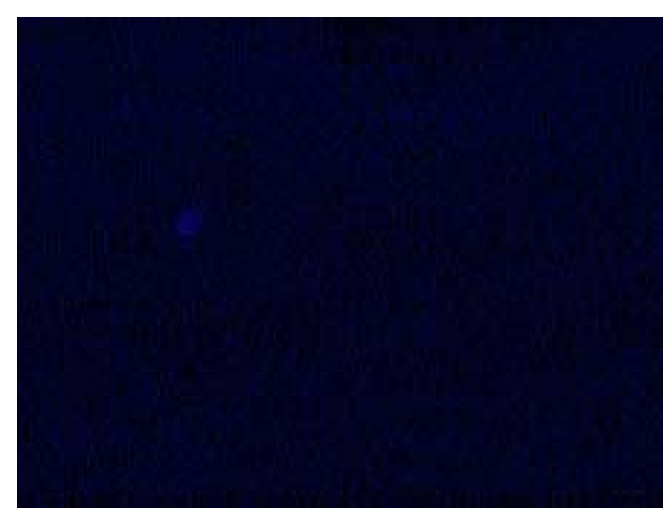

(d)

Figure 4. RIM composite structure thermal images.

$$
\begin{aligned}
& \text { RMSE }=\left[\begin{array}{cccc}
0 & 9.85 & 50.1 & 51.7 \\
0 & 37.8 & 8.65 & 38.9 \\
0 & 33.6 & 9.2 & 11.8
\end{array}\right] \\
& \text { RASE }=\left[\begin{array}{cccc}
0 & 23.8 & 120.8 & 124.7 \\
0 & 103.5 & 23.7 & 106.6 \\
0 & 99.3 & 27.1 & 34.8
\end{array}\right] \\
& \text { Deviation }=\left[\begin{array}{cccc}
0 & 0.05 & 0.97 & 0.99 \\
0 & 0.95 & 0.16 & 0.97 \\
0 & 0.93 & 0.23 & 0.5
\end{array}\right] \\
& \text { Correlation }=\left[\begin{array}{cccc}
1 & 0.96 & 0.28 & 0.13 \\
1 & 0.26 & 0.89 & 0.22 \\
1 & 0.35 & 0.80 & 0.64
\end{array}\right] \\
& \text { Characteristic }=\left[\begin{array}{cccc}
9.85 & 50.1 & 51.7 \\
23.8 & 120.8 & 124.9 \\
0.05 & 0.97 & 0.99 \\
0.96 & 0.28 & 0.13
\end{array}\right]
\end{aligned}
$$

Figures 5(a)-(d) show PVT images for impact damaged GMT composite structure, with Matrices (6)-(9) showing the computed hyperspectral image indices.

$$
\begin{aligned}
& \text { RMSE }=\left[\begin{array}{rrrr}
0 & 57.5 & 67.4 & 68.6 \\
0 & 41.2 & 46.6 & 47.6 \\
0 & 37.9 & 36.8 & 38.6
\end{array}\right] \\
& \operatorname{RASE}=\left[\begin{array}{rrrr}
0 & 88.1 & 103.3 & 105.1 \\
0 & 79.3 & 89.6 & 91.6 \\
0 & 90.2 & 87.7 & 92.0
\end{array}\right] \\
& \text { Deviation }=\left[\begin{array}{llll}
0 & 0.71 & 0.79 & 0.80 \\
0 & 0.54 & 0.72 & 0.73 \\
0 & 0.45 & 0.62 & 0.63
\end{array}\right] \\
& \text { Correlation }=\left[\begin{array}{llll}
1 & 0.56 & 0.35 & 0.32 \\
1 & 0.64 & 0.51 & 0.47 \\
1 & 0.63 & 0.57 & 0.52
\end{array}\right]
\end{aligned}
$$

From Matrices (6)-(9), and with reference to the mentioned criteria of band selection, it is notices that band 3 in (6) has a characteristic for its RMSE values that does not conform to the established criteria, hence band 3 is eliminated. From (9), it is realized that the highest correlation value is in band 2, so band 1 is eliminated, and hence band 2 is selected, which results in the characteristic matrix in (10).

$$
\text { Characteristic }=\left[\begin{array}{ccc}
41.2 & 46.6 & 47.6 \\
79.3 & 89.6 & 91.6 \\
0.54 & 0.72 & 0.73 \\
0.64 & 0.51 & 0.47
\end{array}\right]
$$




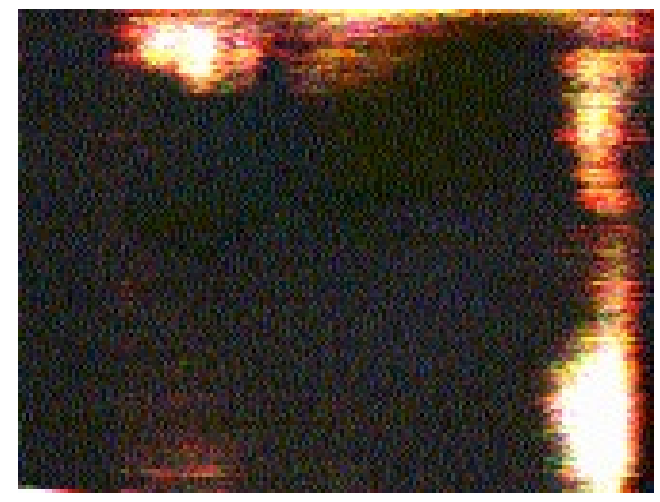

(a)

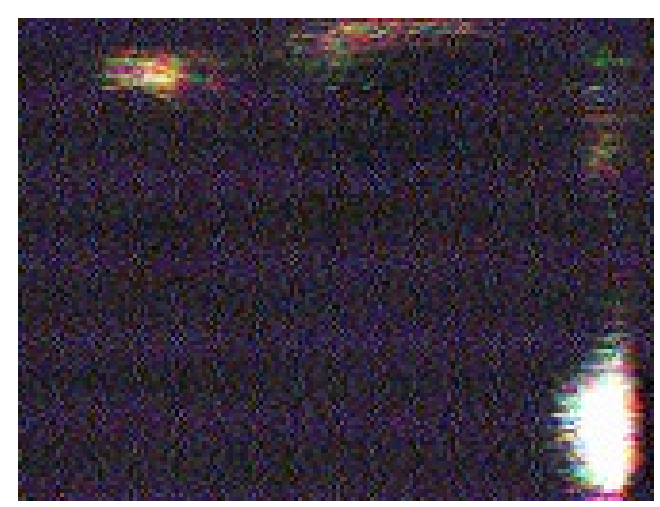

(b)

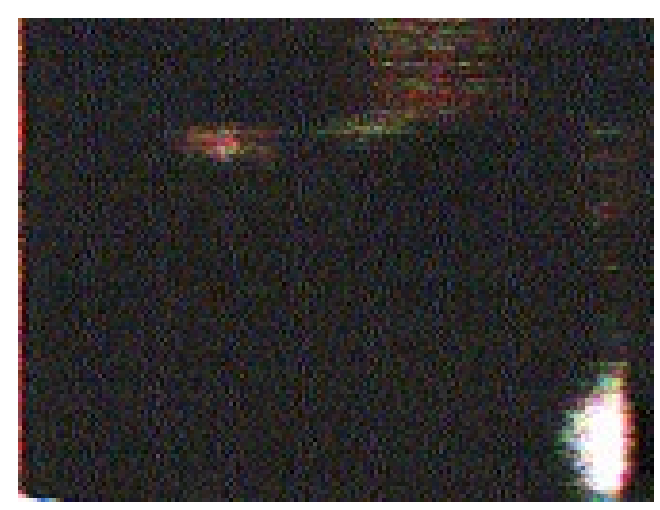

(c)

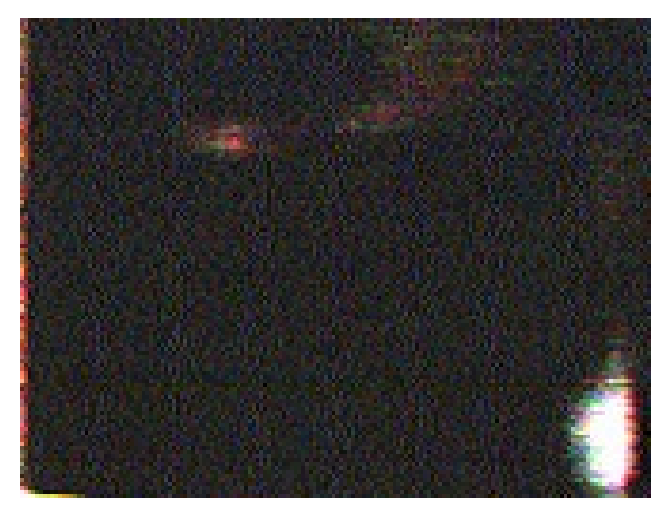

(d)

Figure 5. PVT images for impact damaged GMT composite structure.
The proposed Band elimination technique from a hyperspectral matrix, allows the selection of the best fit image interpreted data with much better accuracy and better damage detection and component condition change. It is also consistent with the general solution of heat wave equation with similar boundary conditions.

From (5) and (10), it is proved that the two tested components are of different type and material as each has different characteristic matrix with different hyperspectral band.

Figures 6-8 show comparisons between the two composite samples.

\section{Conclusion}

Using hyperspectral image analysis technique with band elimination is an effective method to evaluate composite structures and other structures non-destructively using PVT and other non-contactable techniques. As hyperspectral uses different wavelengths to obtain an image signals that subsequently transformed into data, it provides a comprehensive data well for damage detection

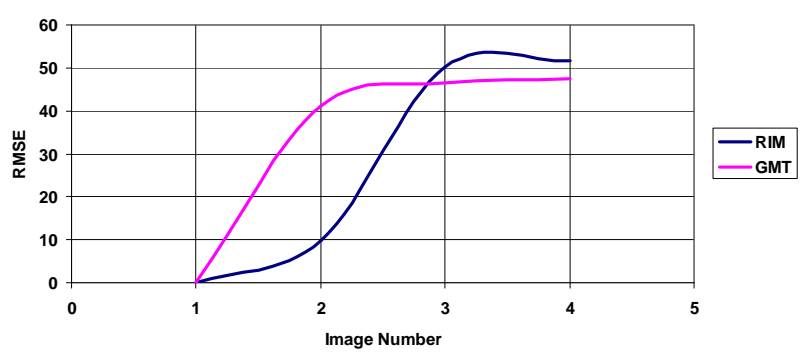

Figure 6. Comparison between GMT and RIM RMSE.

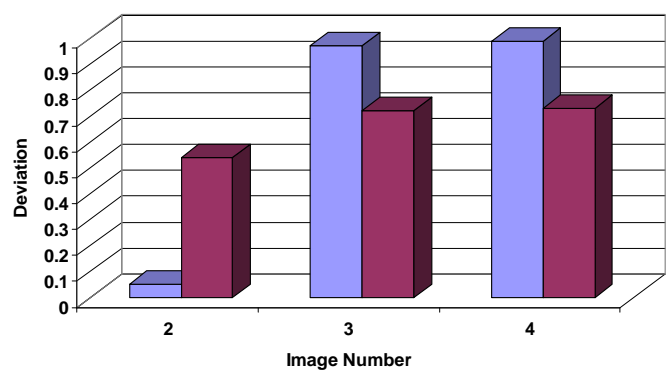

$\square$ RIM

$\square$ GMT

Figure 7. Comparison between Deviation of GMT and RIM Composites.

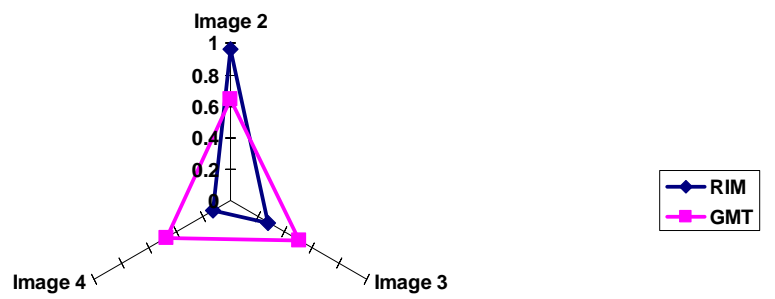

Figure 8. Classification of GMT and RIM using correlation image index. 
and classification. This work proved that testing different types of impact damaged composites impacted using the same equipment and tested using the same PVT system, is not a condition for selecting the same band in the image indices computed for the obtained images. This gives a new angle and a better classification approach to composite structures. Also, using a hyperspectral approach increases the chances of damage detection as there is a band selection algorithm in use. Thus, if the damage is not detected using one band, another band will uncover its presence and effect on the overall performance of the sample.

\section{REFERENCES}

[1] D. P. Myriounis, E. Z. Kordatos, S. T. Hasan and T. E. Matikas, "Crack-Tip Stress Field and Fatigue Crack Growth Monitoring Using Infrared Lock-In Thermography in A359/SiCp Composites," Strain, Vol. 47, No. s1, 2011, pp. e619-e627. doi:10.1111/j.1475-1305.2009.00665.x

[2] B. B. Lahiria, S. Bagavathiappana, P. R. Reshmib, John Philipa, T. Jayakumara and B. Raja, "Quantification of Defects in Composites and Rubber Materials Using Active Thermography," Infrared Physics \& Technology, Vol. 55, No. 2-3, 2012, pp. 191-199. doi:10.1016/j.infrared.2012.01.001

[3] M. Naderi, A. Kahirdeh and M. M. Khonsari, "Dissipated Thermal Energy and Damage Evolution of Glass/Epoxy Using Infrared Thermography and Acoustic Emission," Composites Part B: Engineering, Vol. 43, No. 3, 2012, pp. 1613-1620.

[4] C. Q. Wu, W. P. Wang, Q. G. Yuan, Y. J. Li, W. Zhang and X. D. Zhang, "Infrared Thermography Non-Destructive Testing of Composite Materials," Advanced Materials Research, Vol. 291-294, 2011, pp. 1307-1310. doi:10.4028/www.scientific.net/AMR.291-294.1307

[5] L. Cheng and G. Y. Tian, "Comparison of Nondestructive Testing Methods on Detection of Delaminations in Composites," Journal of Sensors, Vol. 2012, No. 2012, 2012, Article ID: 408437.

[6] M. Kutin, S. Ristić, M. Puharić, M. Vilotijević and M. Krmar, "Thermographic Testing of Epoxy-Glass Composite Tensile Properties," Contemporary Materials, Vol. II, No. 2, 2011, pp. 88-93.

[7] P. Baranowski, W. Mazurek, J. Wozniak and U. Majewska, "Detection of Early Bruises in Apples Using Hyperspectral Data and Thermal Imaging," Journal of Food Engineering, Vol. 110, No. 3, 2012, pp. 345-355. doi:10.1016/j.jfoodeng.2011.12.038

[8] F. D. van der Meer, H. A. van der Werff, F. A. van Ruitenbeek, C. A. Hecker, W. H. Bakker, M. F. Noomen, M. van der Meijde, E. M. Carranza, J. Boudewijn de Smeth and T. Woldai, "Multi- and Hyperspectral Geologic Remote Sensing: A Review," International Journal of Applied Earth Observation and Geoinformation, Vol. 14, No. 1, 2012, pp. 112-128. doi:10.1016/j.jag.2011.08.002

[9] F. Liu, F. Seinstra and A. Plazac, "Parallel Hyperspectral Image Processing on Distributed Multicluster Systems," Journal of Applied Remote Sensing, Vol. 5, No. 1, 2011, pp. 1-14.

[10] A. Picón, O. Ghita, A. Bereciartua, J. Echazarra, P. Whelan and P. Iriondo, "Real-Time Hyperspectral Processing for Automatic Nonferrous Material Sorting," Journal of Electronic Imaging, Vol. 21, No. 1, 2012, pp. 1-9.

[11] Y. Zhao, J. Yang, Q. Zhang, L. Song, Y. Cheng and Q. Pan, "Hyperspectral Imagery Super-Resolution by Sparse Representation and Spectral Regularization," EURASIP Journal on Advances in Signal Processing, Vol. 2011, 2011, p. 87.

[12] R. Darvishzadeha, C. Atzbergerb, A. Skidmorec and M. Schlerfc, "Mapping Grassland Leaf Area Index with Airborne Hyperspectral Imagery: A Comparison Study of Statistical Approaches and Inversion of Radiative Transfer Models," ISPRS Journal of Photogrammetry and Remote Sensing, Vol. 66, No. 6, 2011, pp. 894-906. doi:10.1016/j.isprsiprs.2011.09.013

[13] I. Amro, J. Mateos, M. Vega, R. Molina and A. Katsaggelos, "A Survey of Classical Methods and New Trends in Pansharpening of Hyperspectral Images," EURASIP Journal on Advances in Signal Processing, Vol. 2011, 2011, p. 79.

[14] B. Aiazzi, L. Alparone, S. Baronti, C. Lastri and M. Selva1, "Spectral Distortion in Lossy Compression of Hyperspectral Data," Journal of Electrical and Computer Engineering, Vol. 2012, No. 2012, 2012, Article ID: 850637.

[15] A. Mahyari and M. Yazdi, "Panchromatic and Hyperspectral Image Fusion Based on Maximization of Both Spectral and Spatial Similarities," IEEE Transactions on Geoscience and Remote Sensing, Vol. 49, No. 6, 2011, pp. 1976-1985. doi:10.1109/TGRS.2010.2103944

[16] G. Camps-Valls, J. Benediktsson, L. Bruzzone and J. Chanussot, "Introduction to the Issue on Advances in Remote Sensing Image Processing," IEEE Journal of Selected Topics in Signal Processing, Vol. 5, No. 3, 2011, pp. 365-369. doi:10.1109/JSTSP.2011.2142490 